\title{
Review and Analysis of Various Image Enhancement Techniques
}

\author{
Sandaldeep Kaur \\ Dept of CSE \\ Guru Nanak Dev University Amritsar, \\ India
}

\author{
Prabhpreet Kaur \\ Dept of CSE \\ Guru Nanak Dev University Amritsar \\ India
}

\begin{abstract}
Image enhancement plays an important role in vision applications. Recently a lot of work has been performed in the field of image enhancement. Many techniques have already been proposed till now for enhancing the digital images. This paper has presented a comparative analysis of various image enhancement techniques. This paper has shown that the fuzzy logic and histogram based techniques have quite effective results over the available techniques. This paper ends up with suitable future directions to enhance fuzzy based image enhancement technique further. In the proposed technique, an approach is made to enhance the images other than low-contrast images as well by balancing the stretching parameter $(\mathrm{K})$ according to the color contrast. Proposed technique is designed to restore the degraded edges resulted due to contrast enhancement as well.
\end{abstract}

Keywords: Fuzzy Logic; image processing; color image enhancement; histogram equalization; edge restoration.

\section{INTRODUCTION}

An image is a two dimensional light intensity function $\mathrm{f}(\mathrm{x}, \mathrm{y})$, where $\mathrm{x}$ and $\mathrm{y}$ denotes the spatial co-ordinates and the value of ' $\mathrm{f}$ ' at any point is directly proportional to the brightness(gray level) of the image at that point [1]. Digital image processing is converting an image into its modified better version. In computer science, image processing is any form of signal processing for which the input is an image or frames of videos and output can be either an image or set of parameters related to the image [1]. Image processing is the process of improving image or its features to get maximum details and highlight the useful information. The area under applications of image processing has been increased tremendously. Basic applications of image processing are:

1. Improving the visual quality of images to the next level.

2. Preparing images for extraction of maximum possible features.

Image enhancement is basically improving the interpretability or perception of information in images for human viewers and providing 'better' input for other automated image processing techniques. Main motive behind image enhancement is to modify the attributes of given image to make it suitable for the given task and observer. The modification process may vary according to the given task. Also, more than one attributes of the image can be modified as per the requirements. Various techniques exist for image enhancement and their selection may vary according to the observer-specific factors i.e. humans' visual system and their experience can add great deal of subjectivity to the selection procedure. For visual perception, color images provide more information than gray images. Color image enhancement plays an important role in Digital Image Processing [1]. In color images, poor illumination may result in dark or low contrast images. So such images require enhancement to extract maximum information. In the literature various enhancement techniques such as histogram equalization have been discussed. Contrast enhancement is the process of enhancing the apparent visual quality of that image as well as the specific image feature for further processing and analysis by a computer vision system [1].

\section{VARIOUS IMAGE ENHANCEMENT TECHNIQUES:}

The image enhancement process consists of various techniques that improve the visual appearance of the given image or convert the input image into better form for better analysis by machines as well as humans. Various enhancement techniques are as follows:

1. Spatial domain methods.

2. Frequency domain methods.

3. Fuzzy domain methods.

\section{Spatial Domain Method (SDM):}

Image processing techniques based on spatial methods operate directly on pixels. These methods modify the pixel values according to rules depending on original pixel value i.e. local or point process. Numerous methods exist to compare or combine the pixel values with their immediate or neighboring pixels.

Consider the original image $\mathrm{f}(\mathrm{x}, \mathrm{y})$, transformation $\mathrm{T}$ can be applied to obtain the resultant or processed image $\mathrm{g}(\mathrm{x}, \mathrm{y})$ as:

$$
g(x, y)=T[f(x, y)]
$$

Operator $\mathrm{T}$ is defined over neighborhood pixels of $(\mathrm{x}, \mathrm{y})$. Operator $\mathrm{T}$ is applied to each pixel $(\mathrm{x}, \mathrm{y})$ to obtain $\mathrm{g}$ output at that point. Various SDM based techniques are discussed below: 


\section{Histogram Equalization (HE):}

It is one of the most popular techniques for contrast enhancement of image. HE is a technique based in spatial domain using histogram of the image [1]. A histogram plots the frequency of gray level, at each pixel of the image, varying from 0 (black) to 255 (white). Histogram is a discrete function given by:

$$
\mathrm{h}(\mathrm{rk})=(\mathrm{nk}) / \mathrm{N}
$$

Where, rk and nk are intensity levels of the pixels,

$\mathrm{N}$ is the number of pixels in the image with intensity resp.

Histogram Equalization is a technique that transforms given histogram of the image by spreading the gray-level clusters over a dynamic range. It remaps the gray level frequency based on a probability distribution of input gray-levels of the original image to histogram with near-to uniform probability density function. This technique redistributes the intensity distribution. Histogram having peak and valleys will have peak and valleys even after equalization but these will be shifted [5]. Histogram Equalization can be classified into two principle categories- global and local histogram equalization. Global histogram equalization (GHE) uses entire input for transformation function of the input histogram. While, Local Histogram Equalization (LHE) uses a sliding window that slides through every pixel or block of pixels sequentially and gray-level mapping is performed on the center pixel of that block only. Another methods based on histograms are Histogram Specification that transforms histogram of one image into the histogram of another image, and Dynamic Histogram Specification works on critical points from the input histogram.

\section{Global Histogram Equalization (GHE):}

In this technique, each pixel of the image is assigned a new intensity value based on previous cumulative density function. To perform GHE, the original histogram of the grayscale image needs to be equalized. GHE accounts the global information. The resultant image of GHE is enhanced in contrast. But, it may have unnatural looks due to overenhancement of brightness. Also, GHE technique is not adaptable to local light conditions.

\section{Local Histogram Equalization (LHE):}

This technique uses sub-blocks of the input image and use these blocks to retrieve their histograms. Histogram equalization is applied to the central pixel of that block by applying Cumulative Density function. The process is repeated for every pixel until the end-pixel is equalized. This technique results in over-enhanced portions. This technique is not adaptable with partial light information. Also, computational costs are high for this technique.

\section{Histogram Specification (HS):}

Under this approach, histogram of input image is transformed into the histogram of another image. This approach is used at the times when output is required to form a specific histogram by achieving highlighted gray-level ranges. This approach allows to obtain the desired output. Using this approach is bit complicated, since it's difficult to specify the output histogram as it varies for all the images.

\section{Dynamic Histogram Specification (DHS):}

In these techniques, some Critical Points (CPs) of the input image are selected. On the basis of CPs and some other variants, a specified histogram is created dynamically. This approach enhances the image, by preserving some of the characteristics of the input image's histogram. But, it does not enhance the overall contrast of the image.

Histogram equalization techniques suffer from mean-shift problem [4]. The mean intensity value of the image is shifted to the middle gray-level of the intensity range. Thus, HE based techniques are not useful in the cases where brightness preservation is required.

\subsection{Frequency domain methods (FDT)}

Frequency domain methods are based on Fourier transform. High-frequency contents in the Fourier transform are responsible for Edges and sharp transitions in an image. Smooth areas of image appear due to low frequency contents of Fourier transform. Enhancement of image $f(x, y)$ is performed by applying frequency domain based on DFT.

\subsection{Fuzzy domain}

Various uncertainties and functions in image processing can be easily applied using fuzzy logic. Fuzzy based image processing is collection of various fuzzy approaches that understand, represent and process the image. Fuzzy approach has three main steps: fuzzification, modification of membership function values, and defuzzification. Some steps of fuzzy reasoning can be such as:

Fuzzification: Input values are compared with the membership function to obtain membership values for each part of image in case of image processing.

Modification of membership function values: The membership values are then combined with the defined fuzzy set operations to get weight of each fuzzy rule.

Defuzzification: The qualified output results are combined to obtain crisp output based on the defined methods.

Rules for Fuzzy Inferencing: 
If $\mathrm{x}$ is $\mathrm{A} 1$ and $\mathrm{y}$ is $\mathrm{B} 1$, Then $\mathrm{z}$ is $\mathrm{C} 1$.

If $\mathrm{x}$ is $\mathrm{A} 2$ and $\mathrm{y}$ is $\mathrm{B} 2$, Then $\mathrm{z}$ is $\mathrm{C} 2$.

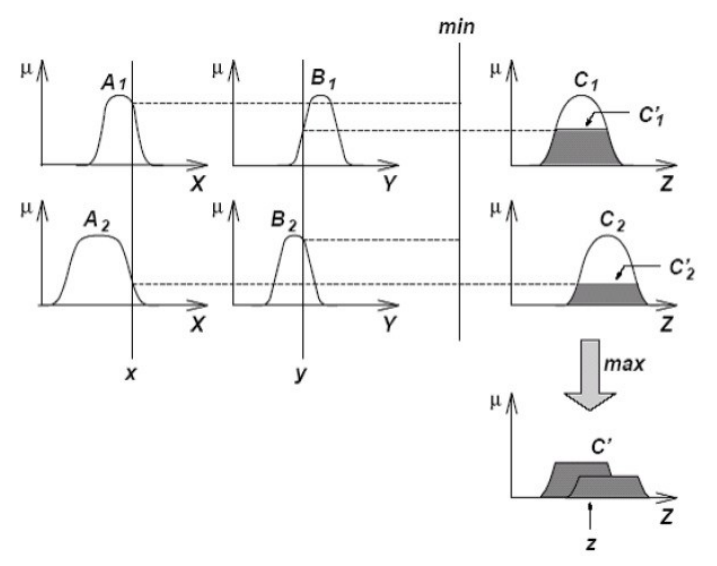

Figure.1 Rules of Fuzzy Reasoning [17]

The process of fuzzification and defuzzification are just the steps for encoding and decoding the image and hence the key point is the modification of membership value. Fuzzy based image processing depends on the fuzzy inference system being used and the input image to be processed.

\section{LITERATURE SURVEY}

Kannan P., Deepa S. and Ramakrishnan [2] presented two approaches for the enhancement of dark sports images. Low contrast images may occur because of poor lightning conditions or small dynamic range of imaging systems. The methods proposed here are fuzzy rule based method and then applying sigmoid functions for the dark and bright inputs. The approach used for enhancement is splitting the color images into RGB planes and applying membership functions to each of the plane. The RGB planes are then concatenated to obtain the resultant enhanced mage. Sigmoid approach is beneficial, since it is flexible; the contrast factors can be adjusted until a satisfactory result is obtained.

K. Hasikin, Ashidi M. I. [3] presented a parameter, named, contrast factor. This parameter provides information on difference among gray-level values in lo9cal neighborhood. It divides the degrade image into bright and dark regions. Gaussian membership function is applied to the respective dark and bright regions separately. For the dark images, sigmoid functions are used to enhance the image. While for colored images, HSV model is used to enhance them. This approach is best applicable for real time applications.

M. Hanmandlu, D. Jha [6] presented a gaussian membership function which fuzzifies the image in spatial domain in order to enhance the given colored image. A global contrast intensification operator (GINT) is introduced which comprises three different parameters namely, intensification parameter, fuzzifier and the crossover point. HSV model is implemented $i$ this paper and the color component is left unmodified. This approach provided a visual improvement to the under-exposed images.

M. Hanmandlu and O.P. Verma [7] proposed a new approach for color image enhancement. An objective function, called exposure is defined to differentiate the underexposed and overexposed regions of image. The image is converted into HSV color space. The hue component $(\mathrm{H})$ is left completely untouched in order to preserve the original colors. For the underexposed images, sigmoid function is used. To recover the lost information in over exposed regions a power law is applied.

O.P. Verma, P. Kumar, M.Hanmandlu [8], enhancement of images over a high range is presented using fuzzy logic and artificial ant colony system. The AACS is used to identify the underexposed, mixed and overexposed regions of an image. The HSV color model is implemented and gaussian factor is used for the fuzzification of over and under-exposed regions, while mixed-exposed regions are left untouched. Parametric sigmoid functions are used for the enhancement. Furthermore, AACS is used to optimize the visual factor of image and thus ascertaining the parametric required for enhancement. The visual appeal is preferred to make the resultant image human eye friendly. This approach is effective in recovering lost information from permanently degraded images.

Preethi S.J., K. Rajeswari [9] presented a membership function ramp used to enhance the visual appearance of the image so hat maximum possible information could be extracted. The membership function is modified for dark and bright regions, but is left unchanged for the middle regions. This approach can be used in medical images to make the diagnosis easy.

O.P. Verma, V.K. Madasu and Shantaram [10] proposed two new transformation functions for the enhancement of under and over-exposed regions of the same image. Rectangular hyperbolic function is used for the under-exposed regions, while for over-exposed regions, S-function is applied. The HSV color model is used for the enhancement purpose. The S-function allows more flexible control for the given regions. The proposed technique is efficient in terms of time required for getting best possible results.

Mahashwari T., Asthana A [11] presented a fuzzy based method for image enhancement. The pixels of image are classified into three classes: dark, bright and gray. On the basis of this classification membership functions are applied by following a global approach. The resultant image obtained is modified and clear.

Shrivastva D., Richhariya V. [12] presented a contrast enhancement technique based on fuzzy entropy principle and fuzzy set theory for low contrast gray scale images. The proposed algorithm is better in contrast enhancement as well as requires less computational time. It is able to overcome the 
drawbacks of spatial domain methods of thresholding. It results in high contrast images.

Chamorro J., Sanchez D. [13] discussed various cardinalities of fuzzy sets and their uses in quantification. The study shows that scalar measures are not well suited for measuring cardinalities and fuzzy numbers suit this well. A new fuzzy based method has been proposed to evaluate the quantified sentences. Linguistic histograms have resulted in more appropriate approach to provide inputs to users. The concepts of color have been defined very well using fuzzy based approach which can be efficiently used in fuzzy image processing.

Raju G. and Madhu S. Nair [14] presented a fast and efficient fuzzy based color image enhancement method for enhancing low contrast color images. This method is based on two parameters $\mathrm{M}$ and $\mathrm{K}$, where $\mathrm{M}$ is average intensity value of image and $\mathrm{K}$ is contrast intensification parameter. The image's RGB factor is converted into HSV color space. To enhance the image, $\mathrm{V}$ factor i.e. intensity is stretched under the control of $\mathrm{M}$ and $\mathrm{K}$. The basic principle on which the technique is designed is transforming skewed histogram of input image into a uniform histogram. The proposed algorithm is compared with conventional techniques. Beside the visual results and computational time, Contrast Improvement Index (CII) and Tenengrad measure are two quantitative measures used for performance analysis. The tenengrad value is larger for high quality images which show that it enhances the structural information, and thus is the result obtained after applying this approach to images. The proposed method is computationally faster than the existing methods and well suits with the images having background with non-uniform distribution of brightness.

Chi-FarnChen, Hung-Yu Chang, Li-Yu Chang [15] presented fuzzy-based approach to enhance the contrast and brightness information on the image. The test results indicated that the proposed method provides better contrast image compared to the conventional enhancement methods in terms of visibility and image details. Two image quality indices have been used to evaluate the performance of the proposed enhancement technique. The comparison of proposed technique with conventional enhancement techniques showed that the proposed method can produce better measurements compared to the conventional techniques. The stretch method used to enhance each cluster is generated by way of a linear model with stretch parameters.

\section{GAPS IN LITERATURE:}

The existing contrast intensification parameter $(\mathrm{K})$, has been taken 128 by most of the researchers, which is only feasible for very low contrast images' enhancement and hence overcontrast images when enhanced result in loss of information. The traditional methods even lay no attention to the regions or objects present in image and enhancement is performed by predefined rules thus resulting in color imbalance of the output image. Conventional techniques also result in images with degraded edges. Since, edges play a significant role in extracting information from images, proposed technique will concentrate on edge restoration as well.

\section{Conclusion:}

This paper has presented a study on various image enhancement techniques. The review has shown that there are still many improvements required in the available techniques to handle different kind of images. This paper has shown that no particular technique is effective for every kind of images or image data set. Although fuzzy logic and histogram based techniques have shown quite significant results but it still face many issues. To overcome the limitations of existing techniques a new technique will be proposed in near future which will evaluate $\mathrm{K}$ factor of fuzzy based enhancement automatically using the ant colony optimization to find the best similarity value among the given set of values which represents the image in more efficient manner.

\section{REFERENCES:}

[1] Gonzalez RC, Woods RE. 2002. Digital image processing. 2nd ed. Englewood Cliffs, NJ: Prentice-Hall. ISBN: 0-20118075-8.

[2] Kannan, P., S. Deepa, and R. Ramakrishnan. 2012. "Contrast enhancement of sports images using two comparative approaches." American Journal of Intelligent Systems 2.6: 141-147.

[3] Hasikin, Khairunnisa, and NorAshidi Mat Isa. 2013. "Adaptive fuzzy intensity measure enhancement technique for non-uniform illumination and low-contrast images." Signal, Image and Video Processing: 1-24.

[4] Kim, Yeong-Taeg. 1997. "Contrast enhancement using brightness preserving bi-histogram equalization." Consumer Electronics, IEEE Transactions on 43.1: 1-8.

[5] Arici, Tarik, SalihDikbas, and YucelAltunbasak. 2009. "A histogram modification framework and its application for image contrast enhancement." Image processing, IEEE Transactions on 18.9: 1921-1935.

[6] Hanmandlu, Madasu, and DevendraJha. 2006. "An optimal fuzzy system for color image enhancement." Image Processing, IEEE Transactions on 15.10 : 2956-2966. 
[7] Hanmandlu, Madasu, et al. 2009. "A novel optimal fuzzy system for color image enhancement using bacterial foraging." Instrumentation and Measurement, IEEE Transactions on $58.8: 2867-2879$.

[8] Verma, Om Prakash, et al. 2012. "High dynamic range optimal fuzzy color image enhancement using artificial ant colony system." Applied Soft Computing 12.1 : 394-404.

[9] SJ, MrsPreethi, and Mrs K. Rajeswari. "Membership Function modification for Image Enhancement using fuzzy logic."

[10] Verma, Om Prakash, V. K. Madasu, and V. Shantaram. 2011. "High Dynamic Range Color Image Enhancement Using Fuzzy Logic and Bacterial Foraging." Defence Science Journal $61.5: 462-472$.

[11] Mahashwari, Tarun, and Amit Asthana. 2013. "Image enhancement using fuzzy technique." International Journal of Research in Engineering Science and Technology $2.2: 1-4$.

[12] Shrivastava, Diwakar, and VineetRichhariya, "Analytical Survey on various parameters."

[13] Chamorro-Martínez, J., et al. 2014. "A discussion on fuzzy cardinality and quantification. Some applications in image processing." Fuzzy Sets and Systems 257 : 85-101.

[14] Raju G., and Madhu S. Nair. 2014. "A fast and efficient color image enhancement method based on fuzzy-logic and histogram." AEU-International Journal of Electronics and Communications $68.3: 237-243$.

[15] Chen, Chi-Farn, Hung-Yu Chang, and Li-Yu Chang. 2008. "A Fuzzy-based method for remote sensing image contrast enhancement." The International Archives of the Photogrammetry, Remote Sensing and Spatial Information Sciences 37 :995-998.

[16] Zadeh, Lotfi A. 1975. "The concept of a linguistic variable and its application to approximate reasoning-II." Information sciences $8.4: 301-357$.

[17] Tizhoosh 1997. "Contrast Improvement based on Fuzzy If-Then rules.

[18] Tizhoosh, Hamid R. 1997. "Fuzzy image processing." Publisher: Springer-Verlag. Kartoniert (TB), Deutsch. 